Algebraic 8 Geometric $\mathcal{T}$ opology

Volume 5 (2005) 1711-1718

Published: 7 December 2005

ATG

\title{
Extensions of maps to the projective plane
}

\author{
JERZY DYDAK \\ MiCHAEL LEVIN
}

\begin{abstract}
It is proved that for a 3 -dimensional compact metrizable space $X$ the infinite real projective space $\mathbb{R} P^{\infty}$ is an absolute extensor of $X$ if and only if the real projective plane $\mathbb{R} P^{2}$ is an absolute extensor of $X$ (see Theorems 1.2 and 1.5).
\end{abstract}

AMS Classification 55M10; 54F45

Keywords Cohomological and extensional dimensions, projective spaces

\section{Introduction}

Let $X$ be a compactum (= separable metric space) and let $K$ be a CW complex. $K \in A E(X)$ (read: $K$ is an absolute extensor of $X$ ) or $X \tau K$ means that every map $f: A \longrightarrow K, A$ closed in $X$, extends over $X$. The extensional dimension $\operatorname{edim} X$ of $X$ is said to be dominated by a CW-complex $K$, written edim $X \leq K$, if $X \tau K$. Thus for the covering dimension $\operatorname{dim} X$ of $X$ the condition $\operatorname{dim} X \leq n$ is equivalent to edim $X \leq S^{n}$ where $S^{n}$ is an $n$-dimensional sphere and for the cohomological dimension $\operatorname{dim}_{G} X$ of $X$ with respect to an abelian group $G$, the condition $\operatorname{dim}_{G} X \leq n$ is equivalent to edim $X \leq K(G, n)$ where $K(G, n)$ is an Eilenberg-Mac Lane complex of type $(G, n)$.

Every time the coefficient group in homology is not explicitely stated, we mean it to be integers.

In case of CW complexes $K$ one can often reduce the relation edim $X \leq K$ to $\operatorname{edim} X \leq K^{(n)}$, where $K^{(n)}$ is the $n$-skeleton of $K$.

Proposition 1.1 Suppose $X$ is a compactum and $K$ is a $C W$ complex. If $\operatorname{dim} X \leq n$, then $\operatorname{edim} X \leq K$ is equivalent to $\operatorname{edim} X \leq K^{(n)}$.

The proof follows easily using edim $X \leq n$ to push maps off higher cells.

Dranishnikov [4] proved the following important theorems connecting extensional and cohomological dimensions. 
Theorem 1.2 Let $K$ be a $C W$-complex and let a compactum $X$ be such that $\operatorname{edim} X \leq K$. Then $\operatorname{dim}_{H_{n}(K)} X \leq n$ for every $n>0$.

Theorem 1.3 Let $K$ be a simply connected $C W$-complex and let a compactum $X$ be finite dimensional. If $\operatorname{dim}_{H_{n}(K)} X \leq n$ for every $n>0$, then $\operatorname{edim} X \leq K$.

The requirement in Theorem 1.3 that $X$ is finite dimensional cannot be omitted. To show that take the famous infinite-dimensional compactum $X$ of Dranishnikov with $\operatorname{dim}_{\mathbb{Z}} X=3$ as in 3 . Then the conclusion of Theorem 1.3 does not hold for $K=S^{3}$. Let us mention in this connection another result [6]: there is a compactum $X$ satisfying the following conditions:

(a) $\operatorname{edim} X>K$ for every finite CW-complex $K$ with $\tilde{H}_{*}(K) \neq 0$,

(b) $\operatorname{dim}_{G} X \leq 2$ for every abelian group $G$,

(c) $\operatorname{dim}_{G} X \leq 1$ for every finite abelian group $G$.

Here $\operatorname{edim} X>K$ means that $\operatorname{edim} X \leq K$ is false.

With no restriction on $K$, Theorem 1.3 does not hold. Indeed, the conclusion of Theorem 1.3 is not satisfied if $K$ is a non-contractible acyclic CW-complex and $X$ is the 2-dimensional disk. Cencelj and Dranishnikov 2] generalized Theorem 1.3 for nilpotent CW-complexes $K$ (see [1] for the case of $K$ with fundamental group being finitely generated).

The real projective plane $\mathbb{R} P^{2}$ is the simplest CW-complex not covered by Cencelj-Dranishnikov's result. Thus we arrive at the following well-known open problem in Extension Theory.

Problem 1.4 Let $X$ be a finite dimensional compactum. Does $\operatorname{dim}_{\mathbb{Z}_{2}} X \leq 1$ imply edim $X \leq \mathbb{R} P^{2}$ ? More generally, does $\operatorname{dim}_{\mathbb{Z}_{p}} X \leq 1$ imply edim $X \leq$ $M\left(\mathbb{Z}_{p}, 1\right)$, where $M\left(\mathbb{Z}_{p}, 1\right)$ is a Moore complex of type $\left(\mathbb{Z}_{p}, 1\right)$ ?

It is not difficult to see that this problem can be answered affirmatively if $\operatorname{dim} X \leq 2$ (use 1.1). Sharing a belief that Problem 1.4 has a negative answer in higher dimensions the authors made a few unsuccessful attempts to construct a counterexample in the first non-trivial case $\operatorname{dim} X=3$ and were surprised to discover the following result.

Theorem 1.5 Let $X$ be a compactum of dimension at most three. If $\operatorname{dim}_{\mathbb{Z}_{2}} X$ $\leq 1$, then $\operatorname{edim} X \leq \mathbb{R} P^{2}$. 
Notice (see [5]) that there exist compacta $X$ of dimension 3 such that $\operatorname{dim}_{\mathbb{Z}_{2}} X$ $\leq 1$, so Theorem 1.5 is not vacuous.

This paper is devoted to proving of Theorem [1.5. Theorem [1.5] can be formulated in a slightly different form. Let $X$ be a compactum. Take $\mathbb{R} P^{\infty}$ as an Eilenberg-Mac Lane complex $K\left(\mathbb{Z}_{2}, 1\right)$. Then $\operatorname{dim} X \leq 3$ and $\operatorname{dim}_{\mathbb{Z}_{2}} X \leq 1$ imply edim $X \leq \mathbb{R} P^{3}$. On the other hand by Theorem 1.2 the condition edim $X \leq$ $\mathbb{R} P^{3}$ implies that $\operatorname{dim}_{\mathbb{Z}_{2}} X=\operatorname{dim}_{H_{1}\left(\mathbb{R} P^{3}\right)} X \leq 1, \operatorname{dim}_{\mathbb{Z}} X=\operatorname{dim}_{H_{3}\left(\mathbb{R} P^{3}\right)} X \leq 3$ and by Alexandroff's theorem $\operatorname{dim} X \leq 3$ if $X$ is finite dimensional (note that $H_{k}\left(\mathbb{R} P^{n}\right)=\mathbb{Z}_{2}$ if $1 \leq k<n$ is odd, $H_{k}\left(\mathbb{R} P^{n}\right)=0$ if $1<k \leq n$ is even, and $H_{n}\left(\mathbb{R} P^{n}\right)=\mathbb{Z}$ if $n$ is odd - see p.89 of [7]). Thus Theorem 1.5] is equivalent to the following, more general result.

Theorem 1.6 Let $X$ be a compactum of finite dimension. If $\operatorname{edim} X \leq \mathbb{R} P^{3}$, then $\operatorname{edim} X \leq \mathbb{R} P^{2}$.

We end this section with two questions related to Theorems 1.5 and 1.6.

Question 1.7 Let $X$ be a compactum of dimension at most three. Does $\operatorname{dim}_{\mathbb{Z}_{p}} X \leq 1$ imply $\operatorname{edim} X \leq M\left(\mathbb{Z}_{p}, 1\right) ?$

Question 1.8 Does $\operatorname{edim} X \leq \mathbb{R} P^{3}$ imply edim $X \leq \mathbb{R} P^{2}$ for all, perhaps infinite-dimensional, compacta $X$ ?

\section{Preliminaries}

\section{Maps on projective spaces}

Recall that the real projective $n$-space $\mathbb{R} P^{n}$ is obtained from the $n$-sphere $S^{n}$ by identifying points $x$ and $-x$. The resulting map $p_{n}: S^{n} \longrightarrow \mathbb{R} P^{n}$ is a covering projection and $\mathbb{R} P^{1}$ is homeomorphic to $S^{1}$. By $q_{n}: B^{n} \longrightarrow \mathbb{R} P^{n}$ we denote the quotient map of the unit $n$-ball $B^{n}$ obtained by identifying $B^{n}$ with the upper hemisphere of $S^{n}$. We consider all spheres to be subsets of the infinite-dimensional sphere $S^{\infty}$. Similarly, we consider all projective spaces $\mathbb{R} P^{n}$ to be subsets of the infinite projective space $\mathbb{R} P^{\infty}$. Clearly, there is a universal covering projection $p: S^{\infty} \longrightarrow \mathbb{R} P^{\infty}$. It is known that $\mathbb{R} P^{\infty}$ has a structure of a CW complex making it an Eilenberg-MacLane complex of type $K\left(\mathbb{Z}_{2}, 1\right)$ as $S^{\infty}$ is contractible. 
Proposition 2.1 Any map $f: \mathbb{R} P^{1} \longrightarrow \mathbb{R} P^{2}$ extends to a map $f^{\prime}: \mathbb{R} P^{2} \longrightarrow$ $\mathbb{R} P^{2}$.

Proof It is obvious if $f$ is null-homotopic. Assume that $f$ is not homotopic to a constant map. Since $\pi_{1}\left(\mathbb{R} P^{2}\right)=\mathbb{Z}_{2}$ and $\mathbb{R} P^{1}$ generates $\pi_{1}\left(\mathbb{R} P^{2}\right), f$ is homotopic to the inclusion map of $\mathbb{R} P^{1}$ to $\mathbb{R} P^{2}$. Obviously, that inclusion extends to the identity map of $\mathbb{R} P^{2}$, so $f$ extends over $\mathbb{R} P^{2}$.

Proposition 2.2 If $f: \mathbb{R} P^{2} \longrightarrow \mathbb{R} P^{2}$ induces the zero homomorphism of the fundamental groups, then $f$ extends to a map $f^{\prime}: \mathbb{R} P^{3} \longrightarrow \mathbb{R} P^{2}$.

Proof Since $f$ induces the zero homomorphism of the fundamental group, $f$ can be lifted to $\beta: \mathbb{R} P^{2} \longrightarrow S^{2}$. Since $H_{2}\left(\mathbb{R} P^{2}\right)=0$, the map $\gamma=\left.\beta \circ q_{3}\right|_{\partial B^{3}}$ : $\partial B^{3} \longrightarrow S^{2}$ induces the zero homomorphism $\gamma_{*}: H_{2}\left(\partial B^{3}\right) \longrightarrow H_{2}\left(S^{2}\right)$ and hence $\gamma$ is null-homotopic. Thus $\gamma$ can be extended over $B^{3}$ and this extension induces the corresponding extension of $f$ over $\mathbb{R} P^{3}$.

Proposition 2.3 Let $Y$ be a topological space. A map $f: S^{1} \times \mathbb{R} P^{1} \longrightarrow Y$ extends over $S^{1} \times \mathbb{R} P^{2}$ if and only if the composition $S^{1} \times S^{1} \stackrel{i d \times p_{1}}{\longrightarrow} S^{1} \times \mathbb{R} P^{1} \stackrel{f}{\longrightarrow}$ $Y$ extends over the solid torus $S^{1} \times B^{2}$.

Proof Consider the induced map $f^{\prime}: \mathbb{R} P^{1} \longrightarrow \operatorname{Map}\left(S^{1}, Y\right)$ to the mapping space defined by $f^{\prime}(x)(z)=f(z, x)$ for $x \in \mathbb{R} P^{1}$ and $z \in S^{1}$. $f$ extends over $S^{1} \times \mathbb{R} P^{2}$ if and only if $f^{\prime}$ extends over $\mathbb{R} P^{2}$. Notice that $f^{\prime}$ extends over $\mathbb{R} P^{2}$ if and only if $S^{1} \stackrel{p_{1}}{\longrightarrow} \mathbb{R} P^{1} \stackrel{f^{\prime}}{\longrightarrow} \operatorname{Map}\left(S^{1}, Y\right)$ extends over $B^{2}$ which is the same as to say that $S^{1} \times S^{1} \stackrel{i d \times p_{1}}{\longrightarrow} S^{1} \times \mathbb{R} P^{1} \stackrel{f}{\longrightarrow} Y$ extends over the solid torus $S^{1} \times B^{2}$.

Proposition 2.4 Suppose $(a, b) \in S^{1} \times \mathbb{R} P^{1}$. If $f: S^{1} \times \mathbb{R} P^{1} \longrightarrow \mathbb{R} P^{2}$ is a map such that $f \mid\{a\} \times \mathbb{R} P^{1}$ is null-homotopic and $f \mid S^{1} \times\{b\}$ is not nullhomotopic, then $f$ extends over $S^{1} \times \mathbb{R} P^{2}$.

Proof Assume $f \mid\{a\} \times \mathbb{R} P^{1}$ is constant. In view of 2.3 we need to show that the composition $S^{1} \times S^{1} \stackrel{i d \times p_{1}}{\longrightarrow} S^{1} \times \mathbb{R} P^{1} \stackrel{f}{\longrightarrow} \mathbb{R} P^{2}$ extends over the solid torus $S^{1} \times B^{2}$. Let $D$ be a disk with boundary equal to $\mathbb{R} P^{1}$. Pick $e: I \longrightarrow S^{1}$ identifying 0 and 1 with $a \in S^{1}$. The homotopy $f \circ(e \times i d): I \times \mathbb{R} P^{1} \longrightarrow \mathbb{R} P^{2}$ has a lift $H: I \times \mathbb{R} P^{1} \longrightarrow S^{2}$ such that $\{0\} \times \mathbb{R} P^{1}$ and $\{1\} \times \mathbb{R} P^{1}$ are each mapped to a point and those points are antipodal as $f \mid S^{1} \times\{b\}$ is not nullhomotopic. Therefore $H$ can be extended to $G: \partial(I \times D) \longrightarrow S^{2}$ so that 
$G \mid\{0\} \times D$ and $G \mid\{1\} \times D$ are constant. Fix the orientation of $\partial(I \times D)$ and let $c$ be the degree of $G$. Define $F$ on $I \times S^{1}$ as the composition $G \circ\left(i d \times p_{1}\right)$ and use the orientation on $\partial\left(I \times B^{2}\right)$ induced by that on $\partial(I \times D)$. Define $F$ on $D_{1}=\{1\} \times B^{2}$ as a map with the same value on $\partial D_{1}$ as $G(\partial(\{1\} \times D))$ so that the induced map from $D_{1} /\left(\partial D_{1}\right) \longrightarrow S^{2}$ is of degree $-c$ (the orientation on $D_{1} /\left(\partial D_{1}\right)$ is induced by the orientation of $\left.\partial\left(I \times B^{2}\right)\right)$. The new map is called $F$. Define $F$ on $D_{0}=\{0\} \times B^{2}$ as the map with the same value on $\partial D_{0}$ as $G(\partial(\{0\} \times D))$ so that $F(0, x)=-F(1, x)$ for all $x \in B^{2}$. The cumulative map $F: \partial\left(I \times B^{2}\right) \longrightarrow S^{2}$ is of degree 0 , so it extends to $F^{\prime}: I \times B^{2} \longrightarrow S^{2}$. Notice that $J=p_{2} \circ F^{\prime}: I \times B^{2} \longrightarrow \mathbb{R} P^{2}$ has the property that $J(0, x)=J(1, x)$ for all $x \in B^{2}$. Therefore it induces an extension $S^{1} \times B^{2} \longrightarrow \mathbb{R} P^{2}$ of the composition $S^{1} \times S^{1} \stackrel{i d \times p_{1}}{\longrightarrow} S^{1} \times \mathbb{R} P^{1} \stackrel{f}{\longrightarrow} \mathbb{R} P^{2}$.

\section{The first modification $M_{1}$ of $\mathbb{R} P^{3}$}

Let $B^{3} \subset \mathbb{R}^{3}$ be the unit ball and let $D$ be the 2 -dimensional disk of radius $1 / 3$ lying in the $y z$-coordinate plane and centered at the point $(0,1 / 2,0)$. Denote by $L$ the solid torus obtained by rotating $D$ about the $z$-axis. We consider $L$ with the structure of cartesian product $L=S^{1} \times D$ such that the rotations of $L$ about the $z$-axis correspond to the rotations of $S^{1}$. Think of $S^{1}$ as the circle $x^{2}+y^{2}=1 / 4, z=0$ (the circle traced by the center of $D$ ). Since $L$ is untouched under the quotient map $q_{3}: B^{3} \longrightarrow \mathbb{R} P^{3}$, we may assume $L \subset \mathbb{R} P^{3}$. The first modification $M_{1}$ of $\mathbb{R} P^{3}$ is obtained by removing the interior of $L$ from $\mathbb{R} P^{3}$ and attaching $S^{1} \times \mathbb{R} P^{2}$ via the map $S^{1} \times \mathbb{R} P^{1} \rightarrow \partial L$, where $\mathbb{R} P^{1}$ is identified with $\partial D$. Notice that $\mathbb{R} P^{2}=q_{3}\left(\partial B^{3}\right) \subset M_{1}$.

Proposition 2.5 There is a retraction $r: M_{1} \longrightarrow \mathbb{R} P^{2}$ of the first modification $M_{1}$ of $\mathbb{R} P^{3}$ to the projective plane.

Proof We use the notation that we introduced above defining the first modification of $\mathbb{R} P^{3}$. Let $I$ be the interval of the points of $B^{3}$ lying on the $z$-axis and let $M=\partial B^{3} \cup I \subset B^{3}$. Denote $K=B^{3} \backslash(L \backslash \partial L)$. Consider the group $\Gamma$ of rotations of $\mathbb{R}^{3}$ around the $z$-axis. Note that $L, M$ and $K$ are invariant under rotations in $\Gamma$ and every such rotation induces the corresponding homeomorphism of $\mathbb{R} P^{2}$ which will be called the corresponding rotation of $\mathbb{R} P^{2}$. Recall that $L$ is represented as the product $L=S^{1} \times D$ in such a way that the rotations of $L$ are induced by the rotations of $S^{1}$. Let $\alpha: K \longrightarrow M$ be a retraction which commutes with the rotations in $\Gamma$ (this means that for every rotation $\rho \in \Gamma$ of $\mathbb{R}^{3}$ and $\left.x \in K, \alpha(\rho(x))=\rho(\alpha(x))\right)$. Let $\beta: M \longrightarrow \mathbb{R} P^{2}$ be the extension of $q_{3}$ restricted to $\partial B^{3}$ sending the interval $I$ to the point 
$q_{3}(\partial I)$. Then $\beta$ also commutes with the rotations in $\Gamma$ (this means that for every $x \in M$, every rotation $\rho \in \Gamma$ of $\mathbb{R}^{3}$ and the corresponding rotation $\rho^{\prime}$ of $\left.\mathbb{R} P^{2}, \beta(\rho(x))=\rho^{\prime}(\beta(x))\right)$. Denote $\gamma=\beta \circ \alpha: K \longrightarrow \mathbb{R} P^{2}$. It is easy to see that $\gamma$ commutes with the rotations. Consider $\partial D$ as a subspace $\partial D=\mathbb{R} P^{1} \subset \mathbb{R} P^{2}$ of a projective plane, and consider $\partial L=S^{1} \times \partial D$ as the subset of $T=S^{1} \times \mathbb{R} P^{2}$ induced by the inclusion $\partial D \subset \mathbb{R} P^{2}$. Fix $a$ in $S^{1}$. By Proposition 2.1 extend $\gamma$ restricted to $\{a\} \times \partial D$ to a map $\mu:\{a\} \times R P^{2} \longrightarrow R P^{2}$ and by the rotations of $S^{1}$ and $\mathbb{R} P^{2}$ extend the map $\mu$ to the map $\kappa: T=S^{1} \times \mathbb{R} P^{2} \longrightarrow \mathbb{R} P^{2}$. Note that $\kappa$ agrees with $\gamma$ on $\partial L=S^{1} \times \partial D$ and therefore the maps $\gamma$ and $\kappa$ define the map $\nu: M_{1} \longrightarrow \mathbb{R} P^{2}$ from the first modification of $\mathbb{R} P^{3}$. This map $\nu$ induces the required retraction $r: M_{1} \longrightarrow \mathbb{R} P^{2}$.

\section{The second modification $M_{2}$ of $\mathbb{R} P^{3}$}

Let $B^{3}$ be the unit ball in $\mathbb{R}^{3}$ and let $L \subset B^{3}$ be the subset of $B^{3}$ consisting of the points lying in the cylinder $x^{2}+y^{2} \leq 1 / 4$. Notice that $R=q_{3}(L)$ is a solid torus in $\mathbb{R} P^{3}$ as the map $B^{2} \longrightarrow B^{2}$ sending $z$ to $-z$ is isotopic to the identity. Set $D=R \cap \mathbb{R} P^{2}$. Represent $R$ as $S^{1} \times D$ such that $\{a\} \times D$ is identified with $D$. The second modification $M_{2}$ of $\mathbb{R} P^{3}$ is obtained by removing the interior of $R$ and attaching $S^{1} \times \mathbb{R} P^{2} \cup\{a\} \times \mathbb{R} P^{3}$ via the inclusion $S^{1} \times \partial D \longrightarrow$ $S^{1} \times \mathbb{R} P^{2} \cup\{a\} \times \mathbb{R} P^{3}$, where $\partial D$ is identified with $\mathbb{R} P^{1}$.

Proposition 2.6 Let $M_{2}$ be the second modification of $\mathbb{R} P^{3}$. The inclusion $i: \mathbb{R} P^{2} \cap M_{2} \hookrightarrow \mathbb{R} P^{2}$ extends to a map $g: M_{2} \longrightarrow \mathbb{R} P^{2}$.

Proof We use the notation that we introduced above defining the notion of the second modification of $\mathbb{R} P^{3}$. Denote $H=\mathbb{R} P^{3} \backslash(R \backslash \partial R)$. Since the center of $B^{3}$ does not belong to $q_{3}^{-1}(H)$, the radial projection sends $q_{3}^{-1}(H)$ to $\partial B^{3}$ and hence the radial projection induces the corresponding map $\alpha$ : $H \longrightarrow \mathbb{R} P^{2}$ which extends the map $i$. Recall that $R$ is represented as $S^{1} \times D$. Then $\partial R=S^{1} \times \partial D$. Fix $a \in S^{1}$ and $b \in \partial D$. Notice that $\alpha \mid\{a\} \times \partial D$ is null-homotopic and $\alpha \mid S^{1} \times\{b\}$ is not null-homotopic. By 2.4 one can extend $\alpha \mid \partial R$ over $S^{1} \times \mathbb{R} P^{2}$. Any such extension is null-homotopic when restricted to $\{a\} \times \mathbb{R} P^{1}$, so it can be extended over $\{a\} \times \mathbb{R} P^{3}$ (see [2.2). Pasting all those extensions gives the desired map $g: M_{2} \longrightarrow \mathbb{R} P^{2}$.

\section{Proof of Theorem 1.5}

Lemma 3.1 Suppose $X$ is a compactum of dimension at most three and mod 2 dimension $\operatorname{dim}_{\mathbb{Z}_{2}} X$ of $X$ equals 1 . A map $f: A \longrightarrow S^{1} \times \mathbb{R} P^{2} \cup\{a\} \times \mathbb{R} P^{3}$ 
extends over $X$ if and only if $\pi \circ f$ extends over $X$, where $\pi: S^{1} \times \mathbb{R} P^{2} \cup\{a\} \times$ $\mathbb{R} P^{3} \longrightarrow S^{1}$ is the projection onto the first coordinate.

Proof Only one direction is of interest. Pick an extension $g: X \rightarrow S^{1}$ of $\pi \circ f$. Let $\pi_{2}: S^{1} \times \mathbb{R} P^{3} \longrightarrow \mathbb{R} P^{3}$ be the projection. Since edim $X \leq \mathbb{R} P^{\infty}$ implies $\operatorname{edim} X \leq \mathbb{R} P^{3}$ (see 1.11), the composition $\pi_{2} \circ f$ extends over $X$ to $h: X \longrightarrow$ $\mathbb{R} P^{3}$. The diagonal $G: X \longrightarrow S^{1} \times \mathbb{R} P^{3}$ of $g$ and $h$ can be pushed rel. $A$ to the 3 -skeleton of $S^{1} \times \mathbb{R} P^{3}$ which is exactly $S^{1} \times \mathbb{R} P^{2} \cup\{a\} \times \mathbb{R} P^{3}$ under standard CW structures of $S^{1}$ and $\mathbb{R} P^{3}$. The resulting map $X \longrightarrow S^{1} \times \mathbb{R} P^{2} \cup\{a\} \times \mathbb{R} P^{3}$ is an extension of $f$.

Corollary 3.2 Suppose $X$ is a compactum of dimension at most three and $A$ is a closed subset of $X$. If mod 2 dimension $\operatorname{dim}_{\mathbb{Z}_{2}} X$ of $X$ equals 1 , then any map $f: A \longrightarrow \mathbb{R} P^{1}$ followed by the inclusion $\mathbb{R} P^{1} \longrightarrow \mathbb{R} P^{2}$ extends over $X$.

Proof Let $i: \mathbb{R} P^{1} \longrightarrow \mathbb{R} P^{3}$ be the inclusion. Extend $i \circ f: A \longrightarrow \mathbb{R} P^{3}$ to $G: X \longrightarrow \mathbb{R} P^{3}$. Let $R$ be the solid torus as in the definition of the second modification of $\mathbb{R} P^{3}$. Put $Y=G^{-1}(R)$ and $B=G^{-1}(\partial R)$. The map $g: B \rightarrow$ $\partial R=S^{1} \times \mathbb{R} P^{1}$ induced by $G$ extends to $H: Y \longrightarrow S^{1} \times \mathbb{R} P^{2} \cup\{a\} \times \mathbb{R} P^{3}$ in view of 3.1. Pasting $G \mid\left(X \backslash G^{-1}(\operatorname{int}(R))\right.$ and $H$ results in an extension $F: X \rightarrow M_{2}$ of $f$. By [2.6 the inclusion $\mathbb{R} P^{2} \cap M_{2} \hookrightarrow \mathbb{R} P^{2}$ extends to a map $g: M_{2} \longrightarrow \mathbb{R} P^{2}$. Notice that $g \circ F$ is an extension of $i \circ f$.

Corollary 3.3 Suppose $X$ is a compactum of dimension at most three. If mod 2 dimension $\operatorname{dim}_{\mathbb{Z}_{2}} X$ of $X$ equals 1 , then any map $f: A \longrightarrow \mathbb{R} P^{2}$ followed by the inclusion $\mathbb{R} P^{2} \longrightarrow M_{1}$ from the projective plane to the first modification of $\mathbb{R} P^{3}$ extends over $X$.

Proof Let $i: \mathbb{R} P^{2} \longrightarrow \mathbb{R} P^{3}$ be the inclusion. Extend $i \circ f: A \longrightarrow \mathbb{R} P^{3}$ to $G: X \longrightarrow \mathbb{R} P^{3}$. Let $L$ be the solid torus as in the definition of the first modification of $\mathbb{R} P^{3}$. Put $Y=G^{-1}(L)$ and $B=G^{-1}(\partial L)$. The map $g: B \rightarrow \partial L=S^{1} \times \mathbb{R} P^{1}$ induced by $G$ extends to $H: Y \longrightarrow S^{1} \times \mathbb{R} P^{2}$ in view of 3.2, Pasting $G \mid\left(X \backslash G^{-1}(\operatorname{int}(L))\right)$ and $H$ results in an extension $F: X \rightarrow M_{1}$ of $f$.

Since $\mathbb{R} P^{2}$ is a retract of $M_{1}$ (see 2.5) , Corollary 3.3 does indeed imply Theorem 1.5 . 
Acknowledgement This research was supported by Grant No. 2004047 from the United States-Israel Binational Science Foundation (BSF), Jerusalem, Israel.

\section{References}

[1] M Cencelj, A N Dranishnikov, Extension of maps to nilpotent spaces. II, Topology Appl. 124 (2002) 77-83 MathReview

[2] M Cencelj, A N Dranishnikov, Extension of maps to nilpotent spaces. III, Topology Appl. 153 (2005) 208-212

[3] A N Dranishnikov, On a problem of P S Aleksandrov, Mat. Sb. (N.S.) 135(177) (1988) 551-557, 560 MathReview

[4] A N Dranishnikov, Extension of mappings into CW-complexes, Mat. Sb. 182 (1991) 1300-1310 MathReview

[5] A N Dranishnikov, Basic elements of the cohomological dimension theory of compact metric spaces, Topology Atlas (1999)

[6] M Levin, Some examples in cohomological dimension theory, Pacific J. Math. 202 (2002) 371-378 MathReview

[7] G W Whitehead, Elements of homotopy theory, Graduate Texts in Mathematics 61, Springer-Verlag, New York (1978) MathReview

Department of Mathematics, University of Tennessee

Knoxville, TN 37996-1300, USA

and

Department of Mathematics, Ben Gurion University of the Negev

P.O.B. 653, Be'er Sheva 84105, ISRAEL

Email: dydak@math.utk.edu and mlevine@math.bgu.ac.il

URL: http://www.math.utk.edu/ dydak

Received: 1 June 2005 